\title{
Minimal factorizations of a cycle: a multivariate generating function
}

\author{
Philippe Biane and Matthieu Josuat-Vergès $\|^{\dagger}$
}

Laboratoire d'Informatique Gaspard Monge, Université Paris-Est Marne-la-Vallée, CNRS

\begin{abstract}
It is known that the number of minimal factorizations of the long cycle in the symmetric group into a product of $k$ cycles of given lengths has a very simple formula: it is $n^{k-1}$ where $n$ is the rank of the underlying symmetric group and $k$ is the number of factors. In particular, this is $n^{n-2}$ for transposition factorizations. The goal of this work is to prove a multivariate generalization of this result. As a byproduct, we get a multivariate analog of Postnikov's hook length formula for trees, and a refined enumeration of final chains of noncrossing partitions.

Résumé. On sait que le nombre de factorisations minimales du long cycle dans le groupe symétrique en un produit de $k$ cycles de longueurs données a une formule très simple: c'est $n^{k-1}$ où $n$ est le rang du groupe symétrique. En particulier, c'est $n^{n-2}$ pour les factorisations en transpositions. Le but de ce travail est de prouver une généralisation multivariée de ce résultat. En conséquence, on obtient un analogue multivarié de la formule des équerres de Postnikov sur les arbres, et une énumération raffinée de chaînes finales de partitions non-croisées.
\end{abstract}

Keywords. minimal factorizations, noncrossing partitions, hook formula

\section{Introduction}

Let $c$ be the long cycle $(1,2,3, \ldots, n)$ in the symmetric group $\mathfrak{S}_{n}$. It is elementary to see that at least $n-1$ factors are needed to write $c$ as a product of transpositions, such as $c=(1,2)(2,3) \ldots(n-1, n)$. So, a factorization

$$
c=t_{1} \ldots t_{n-1}
$$

where each $t_{i}$ is a transposition is called minimal. The number of minimal factorizations of the cycle $c$ is $n^{n-2}$, as was first shown by Dénes [4].

One can interpret this result as the counting of the number of maximal chains in the lattice of noncrossing partitions of $[1, n]$, whose definition is recalled in the text below. The interval partitions, consisting of partitions of $[1, n]$ whose parts are intervals, form a sublattice of the noncrossing partitions, isomorphic to the Boolean lattice of subsets of $[1, n-1]$, whose number of maximal chains is easily seen to be $(n-1)$ !. One of the results of this paper is a formula interpolating between these two, namely we give a generating function for maximal chains $\pi_{0}<\pi_{1}<\cdots<\pi_{n-1}$ in the noncrossing partition lattice of the form

$$
\sum_{\pi_{0}, \pi_{1}, \ldots . \pi_{n-1}} \operatorname{wt}\left(\pi_{0}, \pi_{1}, \ldots, \pi_{n-1}\right)=\prod_{i=1}^{n-2}\left(i X_{i}+n-i\right)
$$

\footnotetext{
${ }^{\dagger}$ Supported by ANR CARMA (ANR-12-BS01-0017)

1365-8050 @ 2016 Discrete Mathematics and Theoretical Computer Science (DMTCS), Nancy, France
} 
where the weight wt is a monomial in the $X_{i}$ and is equal to 1 exactly when $\pi_{0}, \pi_{1}, \ldots \pi_{n-1}$ is a maximal chain of interval partitions.

Let $\underline{a}=\left(a_{1}, \ldots, a_{r}\right)$ where $a_{i} \geq 2$. A factorization $c=z_{1} \ldots z_{r}$ where $z_{i}$ is a cycle of length $a_{i}$ is said to be of type $\underline{a}$. This exists only if $\sum_{i=1}^{r}\left(a_{i}-1\right) \geq n-1$, and the factorization is called minimal in case of equality. We only consider minimal factorizations here and assume $\sum_{i=1}^{r}\left(a_{i}-1\right)=n-1$ from now on. The first author [1] showed that the number of minimal factorizations of type $\underline{a}$ is $n^{r-1}$, in particular it only depends on $r$. This was independently obtained by Du and Liu [5] (see also Irving [8], Springer [13] and the far-reaching generalizations by Krattenthaler and Müller [10]).

We denote $\mathcal{M}(\underline{a})$ the set of minimal factorizations of $c$ of type $\underline{a}$. Again one can interpret such factorizations as chains of a certain type in the lattice of noncrossing partitions and extend the definition of the weight wt to obtain the following

Theorem 1 Let $b_{i}=\sum_{j=1}^{i}\left(a_{j}-1\right)$. We have:

$$
\sum_{z_{1} \ldots z_{r} \in \mathcal{M}(\underline{a})} \operatorname{wt}\left(z_{1} \ldots z_{r}\right)=\prod_{i=1}^{r-1}\left(b_{i} X_{i}+n-b_{i}\right) .
$$

In the context of finite Coxeter groups, Dénes' result is a particular case of Deligne's formula [3] that gives the number of reflection factorizations of a Coxeter element. Deligne's formula has also been interpreted as the number of maximal chains in the noncrossing partition lattice [2]. A one parameter refinement of this enumeration has been obtained by the second author in [9], and it is what naturally leads to the definition of the weight used to get the multivariate versions. Our proof is fully combinatorial, using the fact that there is a simple induction on the number of factors (this being the advantage of considering minimal cycle factorizations, and not just transposition factorizations).

Also, in the particular case of transposition factorizations $\left(a_{i}=2\right.$ for all $\left.i\right)$, Theorem 1 is equivalent to a multivariate hook length formula for trees. This will be presented in Section 4

Again in the particular case of transposition factorizations, our result is in fact equivalent to a multivariate enumeration of Cayley trees of Kreweras and Moszkowski [11]. Indeed, our weights can be translated in terms of decreasing edges of trees. More generally, it is possible to adapt the proof in [11] to the case of decreasing edges in cacti [13], thus giving an alternative full proof of Theorem[1] We give some details in Section 6 .

\section{Definitions and preliminaries}

\subsection{Some classes of partitions}

Let $T$ be a finite totally ordered set.

A noncrossing partition of $T$ is a set partition such that there is no $i<j<k<\ell \in T$ with $i$ and $k$ in one block, and $j, \ell$ in another one. The noncrossing partitions of $T$ form a sublattice of the lattice of set partitions, for the refinement order where $\pi \leq \pi^{\prime}$ if each block of $\pi$ is a subset of some block of $\pi^{\prime}$. We denote it by $N C_{T}$, by $\hat{0}$ the partition with $|T|$ blocks which is the smallest element in $N C_{T}$ and by $\hat{1}$ the partition with one block, which is the largest.

An interval partition of $T$ is a set partition whose blocks are intervals i.e. sets of the form

$$
[a, b]=\{t \in T ; a \leq t \leq b\} \text { for } a, b \in T .
$$


The interval partitions form a sublattice $I_{T}$ of $N C_{T}$. Let $t_{m}$ be the maximal element of $T$, to any interval partition one can associate the set of maximal elements of its blocks, this gives a subset of $T$ which contains $t_{m}$ and conversely, any such subset comes from a unique interval partition. Moreover the order on interval partitions corresponds to the reverse inclusion order on subset of $T$ containing $t_{m}$. Taking the intersection with $T \backslash\left\{t_{m}\right\}$ gives an isomorphism between $I_{T}$ and the Boolean lattice of subsets of $T \backslash\left\{t_{m}\right\}$. In particular, the maximal chains in $I_{T}$ are in bijection with permutations of $T \backslash\left\{t_{m}\right\}$.

The blocks of an interval partition are totally ordered by comparing their elements. Given an interval partition $\left(I_{j}\right)_{j \in \mathcal{J}}$ with at least three blocks, with smallest block $I_{0}$ and largest block $I_{t}$, the partition obtained by merging $I_{0}$ and $I_{t}$ will be called a near interval partition. If $T=\{1, \ldots, n\}$ the near interval partitions are the partitions which are not interval partitions, but can be rotated by $i \mapsto i+k \bmod (n)$ for some $k$ to be transformed into an interval partition.

In the sequel we will consider these definitions when $T$ is the set $\{1, \ldots, n\}$ or a subset with the induced order relation.

\subsection{Embedding noncrossing partitions into the symmetric group}

Let $\pi$ be a noncrossing partition of $T$. There exists a unique a permutation $\sigma_{\pi}$ of $T$ whose orbits are the parts of $\pi$ and, if $i_{1}<i_{2}<\cdots<i_{r}$ form a block of $\pi$, then $\sigma_{\pi}\left(i_{k}\right)=i_{k+1} \bmod (r)$. This defines an embedding $\pi \mapsto \sigma_{\pi}$ of $N C_{T}$ into the group $S_{T}$ of permutations of $T$. The image of this embedding can be characterized geometrically. For each permutation $\sigma$ of $T$ let $l(\sigma)=|T|-c(\sigma)$ where $c(\sigma)$ is the number of orbits of $\sigma$ then $l=l(\sigma)$ is the smallest length of a factorization $\sigma=t_{1} \ldots t_{l}$ into a product of transpositions. It follows that $l$ is a length function i.e. $d(\sigma, \tau)=l\left(\sigma \tau^{-1}\right)$ defines a distance $d$ on the group $S_{T}$, the distance in the Cayley graph, with vertex set $S_{T}$, such that $\left(\sigma, \sigma^{\prime}\right)$ is an edge if and only if $\sigma^{-1} \sigma^{\prime}$ is a transposition. Let $C$ be the long cycle of $S_{T}$ which maps each element of $T$ to its successor and the largest element to the smallest one, then a permutation $\sigma$ is of the form $\sigma_{\pi}$ if and only if it lies on a geodesic for $d$ between the identity permutation $i d$ and $C$ that is, if $l(\sigma)+l\left(C \sigma^{-1}\right)=l(C)=|T|-1$. The order relation on $N C_{T}$ can also be characterized geometrically: one has $\pi \leq \pi^{\prime}$ if and only if $l\left(\sigma_{\pi^{\prime}}\right)=l\left(\sigma_{\pi}\right)+l\left(\sigma_{\pi}^{-1} \sigma_{\pi^{\prime}}\right)$ that is, if $\pi$ lies on a geodesic between $i d$ and $\pi^{\prime}$.

The following lemmas follow from the above geometric characterization.

Lemma 2 Let $C=a z b$ be a factorization with $l(C)=l(a)+l(z)+l(b)$ and $z$ is a cycle on the elements $i_{1}<i_{2} \cdots<i_{r}$ then $z\left(i_{k}\right)=i_{k+1} \bmod (r)$ for $k=1, \ldots, r$.

Proof: One has $C=z\left(z^{-1} a z\right) b$ with $l(C)=l(z)+l\left(\left(z^{-1} a z\right)\right)+l(b)$ therefore $z$ is on a geodesic from $i d$ to $C$, so it is of the form $\sigma_{\pi}$.

Lemma 3 Let $C=y z$ be a minimal factorization with a cycle $z$, then $y=\sigma_{\pi}$ where $\pi$ is an interval or near interval partition.

Proof: Follows easily from the previous lemma.

More generally one has:

Lemma 4 Let $\pi$ be a noncrossing partition and $\sigma_{\pi}=\sigma_{\pi^{\prime}} z$ be a minimal factorization with a cycle $z$ on $k$ elements, then $\pi^{\prime}$ is obtained from $\pi$ by splitting a block of $\pi$ into an interval or near interval partition with $k$ blocks. 
It is immediate to check, using the above properties that, if $C=t_{1} \ldots t_{n-1}$ is a minimal factorization into transpositions, then one has $\sigma_{\pi_{0}}=i d, \sigma_{\pi_{1}}=t_{1}, \sigma_{\pi_{2}}=t_{1} t_{2}, \ldots, \sigma_{\pi_{n-1}}=t_{1} \ldots t_{n-1}=C$ where $\left(\pi_{0}, \ldots, \pi_{n-1}\right)$ is a maximal chain in $N C_{T}$.

\subsection{Some classes of chains in $N C_{T}$}

Definition 5 We denote by $\mathcal{N}(\underline{a})$ the set of $(r+1)$-tuple of noncrossing partitions $\left(\pi_{0}, \ldots, \pi_{r}\right)$ such that:

- $\pi_{0}=\hat{0}$ and $\pi_{r}=\hat{1}$,

- $\pi_{i-1}$ is obtained from $\pi_{i}$ by splitting a block $B$ of $\pi$ into $a_{i}$ blocks $B_{1}, \ldots, B_{a_{i}}$, which form either an interval partition or a near interval partition of $B$.

Proposition 6 The map $\left(\pi_{0}, \ldots, \pi_{r}\right) \mapsto\left(\sigma_{\pi_{0}}, \sigma_{\pi_{0}}^{-1} \sigma_{\pi_{1}}, \ldots, \sigma_{\pi_{r-1}}^{-1} \sigma_{\pi_{r}}\right)$ is a bijection from $\mathcal{N}(\underline{a})$ to $\mathcal{M}(\underline{a})$.

Proof: This follows from Lemma 4

Definition 7 For a sequence $\left(\pi_{0}, \ldots, \pi_{r}\right) \in \mathcal{N}(\underline{a})$ we write $\pi_{i-1} \sqsubset \pi_{i}$ in the case where the blocks $B_{1}, \ldots, B_{a_{i}}$ form an interval partition of $B$. The weight of $\Pi=\left(\pi_{0}, \ldots, \pi_{r}\right) \in \mathcal{N}(\underline{a})$ is

$$
\operatorname{wt}(\Pi)=\prod_{\substack{1 \leq i \leq r \\ \pi_{i-1} \not \dot{m}}} X_{i} .
$$

Using the bijection in Proposition 6, this permits to define the weight function on $\mathcal{M}(\underline{a})$ that was used in Equation (1).

\section{Proof of Theorem 1}

Definition 8 For an $r$-tuple $\underline{a}=\left(a_{1}, \ldots, a_{r}\right)$ such that $a_{i} \geq 2$ for all $i$ and satisfying the minimality condition $\sum_{i=1}^{r}\left(a_{i}-1\right)=n-1$, we define

$$
P_{\underline{a}}\left(X_{1}, \ldots, X_{r-1}\right)=\sum_{\Pi \in \mathcal{N}(\underline{a})} \mathrm{wt}(\Pi) .
$$

For such a $r$-tuple $\underline{a}=\left(a_{1}, \ldots, a_{r}\right)$, we also define $\underline{a}^{\prime}=\left(a_{1}, \ldots, a_{r-2}, a_{r-1}+a_{r}-1\right)$.

Note that $\underline{a}^{\prime}$ also satisfies the minimality condition. Our aim is to prove:

\section{Proposition 9}

$$
P_{\underline{a}}\left(X_{1}, \ldots, X_{r-1}\right)=P_{\underline{a}^{\prime}}\left(X_{1}, \ldots, X_{r-2}\right) \times\left(\left(n-a_{r}\right) X_{r-1}+a_{r}\right) .
$$

Indeed, the formula in Theorem 1 immediately follows by induction (it is clear that $P_{(n)}=1$ ). Note that we have $b_{r-1}=n-a_{r}$ from the definition of $b_{i}$ and the condition $\sum_{i=1}^{r}\left(a_{i}-1\right)=n-1$.

In order to prove the previous proposition, we define a map $\Psi: \mathcal{N}(\underline{a}) \rightarrow \mathcal{N}\left(\underline{a}^{\prime}\right)$ such that

$$
\sum_{\Pi \in \Psi^{-1}(\Gamma)} \operatorname{wt}(\Pi)=\operatorname{wt}(\Gamma) \times\left(\left(n-a_{r}\right) X_{r-1}+a_{r}\right)
$$


for any $\Gamma \in \mathcal{N}\left(\underline{a}^{\prime}\right)$, and summing over $\Gamma$ proves $(2)$.

Let $\Pi=\left(\pi_{0}, \ldots, \pi_{r}\right) \in \mathcal{N}(\underline{a})$ and $\Pi^{\prime}=\left(\pi_{0}, \ldots, \pi_{r-2}, \pi_{r}\right)$. Note that $\Pi^{\prime}$ might not be an element of $\mathcal{N}\left(\underline{a}^{\prime}\right)$. In general, $\Psi(\Pi)$ will have the form $\sigma\left(\Pi^{\prime}\right)=\left(\sigma\left(\pi_{0}\right), \ldots, \sigma\left(\pi_{r-2}\right), \sigma\left(\pi_{r}\right)\right)$ for some $\sigma \in \mathfrak{S}_{n}$. Moreover we require that the restriction of $\sigma$ to each block $B$ of $\pi_{r-2}$ is increasing. Indeed, under these conditions we have:

Lemma 10 Let $1 \leq i \leq r-2$, then there holds $\sigma\left(\pi_{i-1}\right) \sqsubset \sigma\left(\pi_{i}\right)$ if and only if $\pi_{i-1} \sqsubset \pi_{i}$.

Proof: The noncrossing partition $\pi_{i-1}$ is obtained from $\pi_{i}$ by splitting a block $B$. Since $\pi_{i}$ is a refinement of $\pi_{r-2}$, we have $B \subset C$ for some $C \in \pi_{r-2}$, so the restriction of $\sigma$ on $B$ is increasing. Since the order is preserved, the condition of being an interval or a near interval partition is preserved too.

If $1 \leq i \leq r-3$, the previous lemma will ensure that $\mathrm{wt}(\Pi)$ and $\mathrm{wt}(\Psi(\Pi))$ both contain, or both don't contain, a factor $X_{i}$.

We will also define a map $\beta: \mathcal{N}(\underline{a}) \rightarrow\{1, \ldots, n\}$ and we prove that $\Pi \mapsto(\Psi(\Pi), \beta(\Pi))$ is a bijection from $\mathcal{N}(\underline{a})$ to $\mathcal{N}\left(\underline{a}^{\prime}\right) \times\{1, \ldots, n\}$. In the pictures, we represent $\beta(\Pi)=i$ by drawing a vertical bar drawn between two integers $i-1$ and $i$ (if $2 \leq i \leq n$ ) or to the left of 1 (if $i=1$ ). See Figure 1 for examples.

To define $\Psi$ and $\beta$, we use some notations to build noncrossing partitions. Let $\pi$ and $\rho$ be noncrossing partitions, and $i, j>0$, then

- $\pi^{[i]}$ is $\pi$ where all labels are shifted up by $i$.

- $\pi \oplus \rho=\pi \cup \rho^{[j]}$ if $\pi \in \mathcal{N C}_{j}$.

- $(i, j) \curvearrowright \pi=\{\{1 \ldots, i\} \cup\{n-j+1, \ldots, n\}\} \cup \pi^{[i]}$.

- when there is no ambiguity, an integer $i$ denote the one block partition of size $i$.

For example, a near interval partition with four blocks can be written $(a, b) \curvearrowright(c \oplus d \oplus e)$ where $a, b, c, d, e>0$.

Let $B$ denote the block of $\pi_{r-1}$ that splits in $\pi_{r-2}$. Moreover, $I, J, K$ denote arbitrary interval partition, and $a, b, c$, etc. are integers (or one-block partitions). Then the definition is the following (see also Figure 11:

- Case 1: $\pi_{r-1}$ and $\pi_{r-2}$ are both interval partitions.

Then $\sigma$ is the identity permutation, and $\beta(\Pi)=\min B$.

- Case 2: $\pi_{r-2}$ is an interval partition but $\pi_{r-1}$ is not.

Then $\sigma$ is the identity permutation, and $\beta(\Pi)=n-b+1$ where $b$ is such that we can write $\pi_{r-1}=(a, b) \curvearrowright I$.

- Case 3: $\pi_{r-1}=(a, b) \curvearrowright(I \oplus c \oplus J)$ and $\pi_{r-2}=(a, b) \curvearrowright(I \oplus K \oplus J)$.

Then $\sigma\left(\pi_{r-2}\right)=I \oplus(a+b) \oplus K \oplus J$. (Although we do not define $\sigma$ explicitly, there is only one canonical choice.) And $\beta(\Pi)=\min B$.

- Case 4: $\pi_{r-1}=(a, b) \curvearrowright I$, and $\pi_{r-2}=J \oplus\left(a^{\prime}, b^{\prime}\right) \curvearrowright I \oplus K$ with $0<a^{\prime} \leq a$ and $0<b^{\prime} \leq b$. Then $\sigma\left(\pi_{r-2}\right)=J \oplus I \oplus\left(a^{\prime}+b^{\prime}\right) \oplus K$, and the bar is placed between the $a^{\prime}$ first dots and $b^{\prime}$ last dots of the block of size $a^{\prime}+b^{\prime}$. 
- Case 5 : $\pi_{r-1}=I \oplus a \oplus J$ and $\pi_{r-2}=I \oplus(b, c) \curvearrowright K \oplus J$.

Then $\sigma\left(\pi_{r-2}\right)=(b, c) \curvearrowright(I \oplus K \oplus J)$. If $I$ is nonempty, the bar is placed to the left of its last block. Otherwise, the bar is placed in leftmost position $(\beta(\Pi)=1)$.

- Case 6: $\pi_{r-1}=(a, b) \curvearrowright I$, and $\pi_{r-2}=\left(a^{\prime}, b^{\prime}\right) \curvearrowright(J \oplus I \oplus K)$ with $0<a^{\prime} \leq a$ and $0<b^{\prime} \leq b$. Then $\sigma$ is the identity, and the bar is placed between $I$ and $K$.

- Case 7: $\pi_{r-1}=(a, b) \curvearrowright(I \oplus c \oplus J)$, and $\pi_{r-2}=(a, b) \curvearrowright(I \oplus(d, e) \curvearrowright K \oplus J)$.

Then $\sigma\left(\pi_{r-2}\right)=(a, b) \curvearrowright(I \oplus(d+e) \oplus J \oplus K)$, and the bar is placed between the $d$ first dots and $e$ last dots of the block of size $d+e$.

- Case 8: $\pi_{r-1}=(a, b) \curvearrowright I$, and $\pi_{r-2}=\left(a^{\prime}, b^{\prime}\right) \curvearrowright(J \oplus(d, e) \curvearrowright I \oplus K)$ with $0<a^{\prime} \leq a$ and $0<b^{\prime} \leq b$.

Then $\sigma\left(\pi_{r-2}\right)=\left(a^{\prime}, b^{\prime}\right) \curvearrowright(J \oplus I \oplus(d+e) \oplus K)$, and the bar is placed between the $d$ first dots and $e$ last dots of the block of size $d+e$.

- Case 9 and 10: This is when $\pi_{r-2}$ contains a block $C$ which is a union of three intervals (and no less). Let $a, b, c$, denote the length of these intervals. The other blocks of $\pi_{r-2}$ are arranged as a union of two interval partitions $I$ and $J$ (from left to right).

If $\pi_{r-1}$ is obtained by joining $C$ with the blocks in $I$ (Case 9), then $\sigma\left(\pi_{r-2}\right)=(a+b, c) \curvearrowright(I \oplus J)$, and the bar is placed to the right of the $a$ th dot.

If $\pi_{r-1}$ is obtained by joining $C$ with the blocks in $J$ (Case 10), then $\sigma\left(\pi_{r-2}\right)=(a, b+c) \curvearrowright$ $(I \oplus J)$, and the bar is placed between to the left of the $c$ th dot starting from the right.

We can check that $\pi_{r-2} \sqsubset \pi_{r-1}$ if and only if $\sigma\left(\pi_{r-2}\right)$ is an interval partition (both conditions are true in cases $1-4$, but none is true in cases 5-10). This means that $\operatorname{wt}(\Pi)$ and $\operatorname{wt}(\Psi(\Pi))$ both contain or don't contain a factor $X_{r-2}$. So at this point, we have proved that $\operatorname{wt}(\Psi(\Pi))=\operatorname{wt}(\Pi) \operatorname{or} \operatorname{wt}(\Psi(\Pi)) \times X_{r-1}=$ $\mathrm{wt}(\Pi)$.

Lemma 11 Suppose we know $\sigma\left(\pi_{r-2}\right)$ and the location of the bar, then we can deduce which one of the 10 cases was applied.

Proof: Suppose for example that $\sigma\left(\pi_{r-2}\right)$ is a near interval partition, and the bar is between $i$ and $i+1$ where both of these integers are in the non-interval block of $\sigma\left(\pi_{r-2}\right)$. By examining the various cases in Figure 1, we see that we are in case 9 or 10 depending on whether the bar is on the left or right part of this non-interval block. It remains only to distinguish cases $1-8$.

- We can separate cases 1-4 from 5-8 by seeing whether $\sigma\left(\pi_{r-2}\right)$ is an interval partition, or not.

- We can separate cases $3,4,7,8$ from cases $1,2,5,6$ by seeing whether the bar is between two integers that are in the same block of $\sigma\left(\pi_{r-2}\right)$, or not.

- We can separate cases $1,3,5,7$ from cases $2,4,6,8$ by seeing whether the number of blocks of $\sigma\left(\pi_{r-2}\right)$ entirely to the right of the bar is $\geq a_{r-1}$ or $<a_{r-1}$. 
Case 1

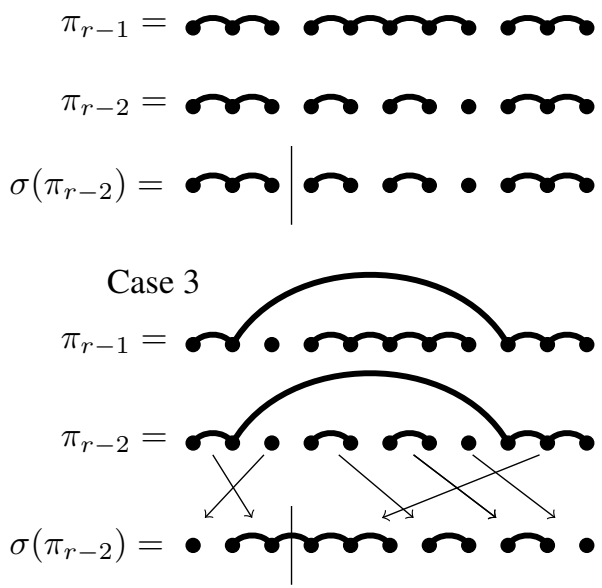

Case 5

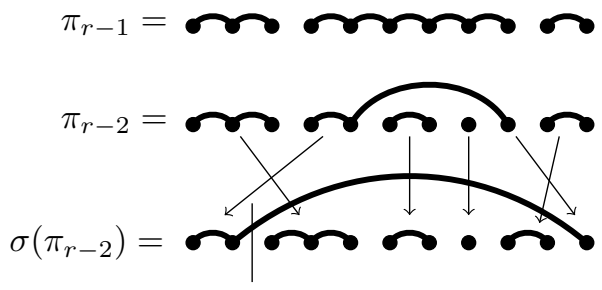

Case 7
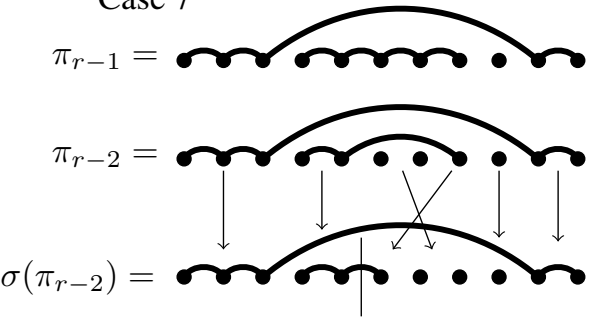

Case 9
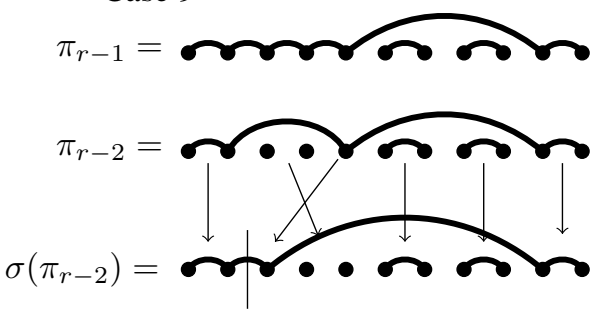

Case 2
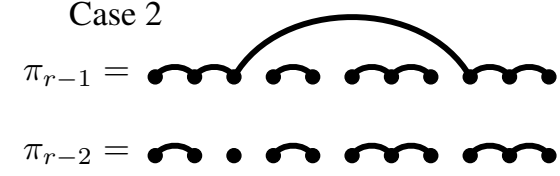

$\sigma\left(\pi_{r-2}\right)=\boldsymbol{r} \boldsymbol{m}$

Case 4
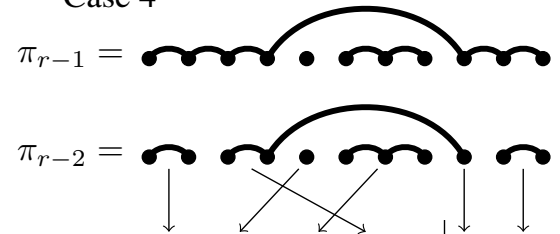

$\sigma\left(\pi_{r-2}\right)=\boldsymbol{r} \boldsymbol{r} \boldsymbol{r}$

Case 6
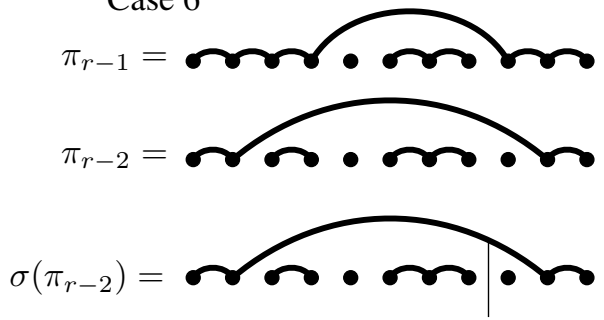

Case 8

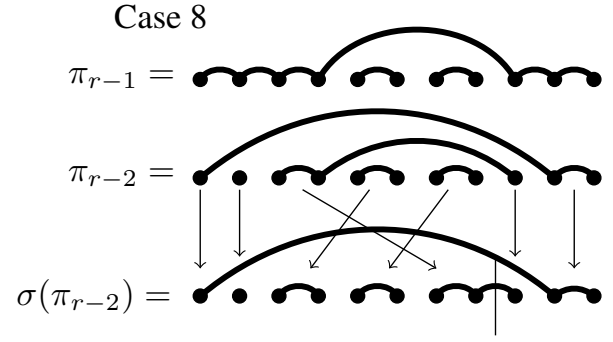

Case 10
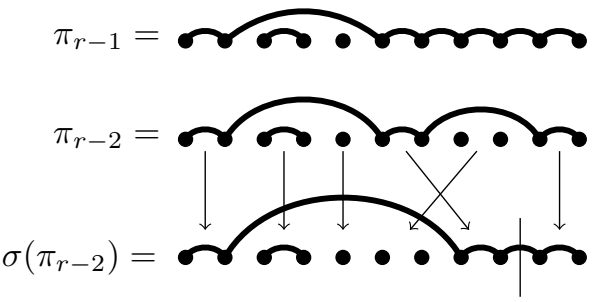

Figure 1: The map $\Psi$. 
So the 3 criterions permits to distinguish the $2^{3}=8$ remaining cases.

The previous lemma implies that $(\Psi, \beta)$ is a bijection, because in each given case we can recover $\pi_{r-2}$ and $\pi_{r-1}$ from $\sigma\left(\pi_{r-2}\right)$ and the location of the bar. So for a given $\Gamma \in \mathcal{N}\left(\underline{a}^{\prime}\right)$, the $n$ elements in $\Psi^{-1}(\Gamma)$ can be obtained from the $n$ possible locations of the bar.

In order to get the factor $\left(\left(n-a_{r}\right) X_{r-1}+a_{r}\right)$ in (3), it remains to prove the following :

Lemma 12 If $\sigma\left(\pi_{r-2}\right)$ is given, among the $n$ possible locations of the bar, there are exactly $a_{r}$ that result in $\pi_{r-1}$ being an interval partition.

Proof: Suppose first that $\sigma\left(\pi_{r-2}\right)$ is an interval partition. The only case where $\pi_{r-1}$ is an interval partition is case 1 . This means that the bar need to be located just to the left of some block, and need to have at least $a_{r-1}$ blocks to its right. Since the number of blocks in $\sigma\left(\pi_{r-2}\right)$ is $a_{r-1}+a_{r}-1$, there are $a_{r}$ possible locations.

Now suppose that $\sigma\left(\pi_{r-2}\right)$ is not an interval partition. The only case where $\pi_{r-1}$ is an interval partition is case 5. Once again this means that the bar need to be located just to the left of some block, and need to have at least $a_{r-1}$ blocks to its right. In this case too we get $a_{r}$ possible locations.

This completes the proof of Equation (3). As explained earlier, we deduce Theorem 1 .

\section{A hook formula for labelled trees}

In this section, we are in the particular case $\underline{a}=(2, \ldots, 2)$ where there are $n$ 2's (this corresponds to counting transposition factorizations in $\left.\mathfrak{S}_{n+1}\right)$. We give a mutivariate version of Postnikov's hook length formula [12, Corollary 17.3] as a consequence of Theorem 11. The interpretation of this hook length formula using noncrossing chains has been made by the second author in [9. Section 5], and along the same lines it gives the multivariate version we present here.

Note that another mutivariate hook length formula generalizing Postnikov's was obtained by Féray and Goulden [6]. It seems unrelated to ours (in the sense that one would easily implies the other), but it is likely that their methods can give another proof of our Theorem 15 below.

Let us define a polynomial

$$
P_{n}\left(X_{0}, \ldots, X_{n-1}\right)=\sum_{\Pi \in \mathcal{N}(\underline{a})} \mathrm{wt}(\Pi) .
$$

By Theorem 1 it is equal to $\prod_{i=1}^{n-1}\left(i X_{i}+(n+1-i)\right)$. Note that $P_{n}$ is considered as an $n$-variable polynomial. Introducing the (seemingly useless) variable $X_{0}$ makes more convenient to write the next lemma.

Lemma 13 Let $n \geq 2$, then we have

$$
P_{n}\left(X_{0}, \ldots, X_{n-1}\right)=\frac{(n-1) X_{n-1}+2}{2} \sum P_{\# I}\left(X_{i_{1}}, X_{i_{2}}, \ldots\right) P_{\# J}\left(X_{j_{1}}, X_{j_{2}}, \ldots\right)
$$

where the sum is over $I, J$ such that $\{0, \ldots, n-2\}=I \uplus J$, and $I=\left\{i_{1}, i_{2}, \ldots\right\}, J=\left\{j_{1}, j_{2}, \ldots\right\}$. This is a recursion whose initial case is $P_{1}\left(X_{0}\right)=1$. 
Proof: Let $\Pi=\left(\pi_{0}, \ldots, \pi_{n}\right) \in \mathcal{N}(\underline{a})$, and denote by $B$ and $C$ the two blocks of $\pi_{n-1}$. Because of the symmetry, it is convenient to say that $B$ is a distinguished block of $\pi_{n-1}$, and after computing the generating function of such objects we divide the result by 2 . Then we consider:

$$
\begin{aligned}
& \Pi_{1}=\left(\left.\pi_{0}\right|_{B}, \ldots,\left.\pi_{n-1}\right|_{B}\right), \\
& \Pi_{2}=\left(\left.\pi_{0}\right|_{C}, \ldots,\left.\pi_{n-1}\right|_{C}\right),
\end{aligned}
$$

where $\left.\pi\right|_{B}=\{X \in \pi: X \subset B\}$. Let $\Pi_{1}^{\prime}$ (respectively, $\Pi_{2}^{\prime}$ ) be what we obtain after removing the repeated entries in $\Pi_{1}$ (respectively, $\Pi_{2}$ ). To encode the location of repeated entries we define:

$$
\begin{aligned}
& I=\left\{i:\left.\pi_{i}\right|_{B}=\left.\pi_{i+1}\right|_{B}\right\}, \\
& J=\left\{i:\left.\pi_{i}\right|_{C}=\left.\pi_{i+1}\right|_{C}\right\}
\end{aligned}
$$

We have $I \uplus J=\{0, \ldots, n-2\}$, moreover $\Pi_{1}^{\prime}$ (respectively $\Pi_{2}^{\prime}$ ) is a maximal chain of $N C_{B}$ (respectively $N C_{C}$ ). These properties follows the fact that the interval $\left[\hat{0}, \pi_{n-1}\right]$ is isomorphic to $N C_{B} \times N C_{C}$.

The map $\Pi \mapsto\left(B, C, I, J, \Pi_{1}, \Pi_{2}\right)$ is bijective and proves combinatorially Equation (4). Indeed, for fixed $B$ and $C$ we get the sum over $I$ and $J$. To get the factor $(n-1) X_{n-1}+2$, observe that when \#I and $\# J$ are fixed, all the possible $B$ and $C$ are obtained from each other by the cyclic rotation through $\{1, \ldots, n+1\}$, and there are two interval partitions in this orbit. It remains only to divide by 2 for symmetry reasons as mentionned above.

The recursion in the previous lemma is conveniently interpreted in terms of trees. See the discussion at the end of this section for more information about this particular kind of trees.

Definition 14 An André tree on $n$ vertices is a labelled tree such that

- each internal vertex has either one or two unordered descendants,

- vertices are labelled with integers from 1 to $n$, decreasingly from the root to the leaves.

We denote $\mathcal{A}_{n}$ the set of André trees with $n$ vertices. If $1 \leq i \leq n$, we denote by $h_{i}(T)$ the hook length of the vertex with label $i$ in $T$, i.e. the number of vertices below the vertex with label $i$ (including itself). The weight of an André tree $T \in \mathcal{T}_{n}$ is defined as

$$
\mathrm{wt}(T)=\prod_{\substack{1 \leq i \leq n \\ h_{i}(T)>1}}\left(X_{i-1}\left(h_{i}(T)-1\right)+2\right) .
$$

For example, the André trees on 4 vertices are in Figure 2

Theorem 15 We have:

$$
\sum_{T \in \mathcal{T}_{n}} \mathrm{wt}(T)=\prod_{i=1}^{n-1}\left(i X_{i}+(n+1-i)\right) .
$$

Proof: We show that the left hand side satisfies the same recursion as $P_{n}$, as given in Lemma 13 . The result is clear for $n=1$, so let $n \geq 2$. 

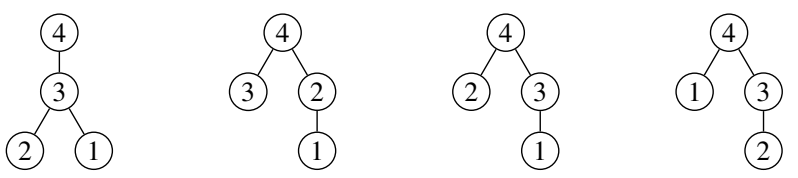

Figure 2: The André trees with 4 vertices.

For each $T \in \mathcal{T}_{n}$, the contribution of the root to $\mathrm{wt}(T)$ is a factor $(n-1) X_{n-1}+2$, since its hook length is $n$. The rest of the tree is an unordered pair $\{U, V\}$ of trees, one of them being possibly empty (in the case where the root has only one descendant). We can rather consider ordered pairs $(U, V)$ and divide the result by 2 at the end, since $U$ and $V$ can always be distinguished by the labels they contain. This gives the factor $\frac{(n-2) X_{n-2}+2}{2}$.

The labels of $U$ and $V$ form a partition $\{1, \ldots, n-2\}=I \uplus J$, and the tree $U$ (respectively, $V$ ) is an element of $\mathcal{A}_{\# I}$ (respectively, $\mathcal{A}_{\# J}$ ) with an appropriate relabelling. This permits to write the recursion. We omit details.

For example, the hook formula with $n=4$ is as follows (where the five terms are in the order coming from the trees in Figure 2):

$$
\begin{aligned}
& \left(2+3 X_{3}\right)\left(2+2 X_{2}\right)\left(2+X_{1}\right)+\left(2+3 X_{3}\right)\left(2+2 X_{2}\right) \\
& +\left(2+3 X_{3}\right)\left(2+X_{1}\right)+\left(2+3 X_{3}\right)\left(2+X_{2}\right)+\left(2+3 X_{3}\right)\left(2+X_{2}\right) \\
= & \left(X_{1}+4\right)\left(2 X_{2}+3\right)\left(3 X_{3}+2\right) .
\end{aligned}
$$

André trees were first introduced by Foata and Schützenberger [7], who showed that $\# \mathcal{A}_{n}$ is the $n$th Euler number $E_{n}$ (which can be defined as the number of alternating permutations in $\mathfrak{S}_{n}$ ). They were used by Stanley [14] to show that $E_{n}$ is the number of orbits for the action of $\mathfrak{S}_{n+1}$ on maximal chains of set partitions on $\{1, \ldots, n+1\}$, so they were not unexpected in the present context. Stanley's bijection explains why proving the above recursion is essentially the same on chains of partitions or on André trees. But it also gives a fully combinatorial interpretation of the hook formula as follows. Although $\mathcal{N}(\underline{a})$ is not stable under the action of $\sigma_{n+1}$, we can consider the equivalence relation $\sim$ on $\mathcal{N}(\underline{a})$ defined by $\Pi_{1} \sim \Pi_{2}$ if there is $\sigma \in \mathfrak{S}_{n}$ such that $\sigma\left(\Pi_{1}\right)=\Pi_{2}$. It is easy to see that each orbit of maximal chains of partitions contains a chain of noncrossing partitions, so the equivalence classes are indexed by André trees. And the generating function of the equivalence class of index $T$ is $\operatorname{wt}(T)$. So the hook express the fact that equivalence classes form a partition of $\mathcal{N}(\underline{a})$.

We end this section by an open question. It would be very interesting if the recursion in Lemma 13 could be solved in a direct way leading to $P_{n}\left(X_{0}, \ldots, X_{n-2}\right)=\prod_{i=1}^{n-2}\left(i X_{i}+(n-i)\right)$. It would give an alternative proof of our multivariate hook formula, or equivalently, of the transposition case of Theorem 1 The methods of [6] are quite likely to apply for this kind of problem.

\section{Final chains of noncrossing partitions}

In this section, we present another interesting consequence of Theorem 1 . We are still in the transposition case $\left(r=n-1\right.$ and $a_{i}=2$ for all $\left.i\right)$. 
Definition 16 A final chain of length $k$ in $N C_{n}$ is a k-tuple of elements $\left(\pi_{n-k}, \ldots, \pi_{n-1}\right)$ such that $\pi_{n-k} \lessdot \cdots \lessdot \pi_{n-1}=\hat{1}$. The weight of such a chain $\Pi=\left(\pi_{n-k}, \ldots, \pi_{n-1}\right)$ is

$$
\operatorname{wt}(\Pi)=\prod_{\substack{n-k \leq i \leq n-2 \\ \pi_{i} \not{\not}}} X_{i} .
$$

It follows from the results of Krattenthaler and Müller [10] that the number of final chains of length $k$ in $N C_{n}$ is $n^{k-2}\left(\begin{array}{l}n \\ k\end{array}\right)$. A multivariate analog can be obtained from Theorem 1 .

Corollary 17 We have

$$
\sum \mathrm{wt}(\Pi)=\frac{1}{n}\left(\begin{array}{l}
n \\
k
\end{array}\right) \prod_{i=n-k}^{n-2}\left(i X_{i}+(n-i)\right)
$$

where the sum is over final chains of length $k$ in $N C_{n}$.

Proof: Let us consider the set $S$ of maximal chains $\pi_{0} \lessdot \cdots \lessdot \pi_{n-1}$ with the property that $\pi_{0} \sqsubset \cdots \sqsubset$ $\pi_{n-k}$. The weight generating function of $S$ is obtained via a specialization of Theorem 1 . more precisely we take $r=n-1, a_{i}=2$ for all $i$, then $X_{i}=0$ for $1 \leq i \leq n-k-1$. This gives $\prod_{i=n-k}^{n}$ - $\left(i X_{i}+(n-\right.$ i)) $\times \prod_{i=k+1}^{n-1} i$.

Given a final chain $\pi_{n-k} \lessdot \cdots \lessdot \pi_{n-1}=\hat{1}$ of length $k$, there are $(n-k)$ ! ways to complete it into a maximal chain in $S$. This is a consequence of Lemma 18 below. So the generating function for final chains of length $k$ is $\frac{1}{(n-k) !}$ times that of $S$. The result follows.

Lemma 18 Let $\pi \in N C_{n}$, and $r$ denote its rank. There are exactly $r$ elements $\rho$ satisfying $\rho \lessdot \pi$ and $\rho \sqsubset \pi$.

\section{Decreasing edges in Cayley trees and cact ${ }^{(i)}$}

It is well known that there are $n^{n-2}$ Cayley trees on $n$ vertices. Each Cayley tree is considered to be rooted at the vertex with label $n$, and edges are oriented towards the root. An edge is decreasing if it is oriented from $i$ to $j$ with $i>j$. The weight of a Cayley tree is a square free monomial in variables $X_{1}, X_{2}, \ldots$ such that there is a factor $X_{j-1}$ iff there is a decreasing edge starting from the vertex with label $j$. The following result comes from [11]:

Theorem 19 The weight generating function of Cayley trees is $\prod_{i=1}^{n-2}\left(i X_{i}+n-i\right)$.

In the same vein, it is possible to give an interpretation of the right-hand side of (1) using cacti (see [13]). These trees and cacti are in bijection with chains of noncrossing partitions, and the factorized generating function can be proved using codes similar to Prüfer codes.

\section{Acknowledgement}

We thank the anonymous reviewer who informed us that our work is related with decreasing edges in Cayley trees and cacti, and in particular gave an alternative proof of our main result. Only parts of this could be reproduced here, in Section 6

(i) The content of this section comes from a reviewer's report. See acknowledgements for details. 


\section{References}

[1] P. Biane: Minimal factorizations of a cycle and central multiplicative functions on the infinite symmetric group. J. Combin. Theory A 76 (1996), 197-212.

[2] F. Chapoton: Enumerative properties of generalized associahedra, Sém. Lothar. Combin. 51 (2004), Article B51b.

[3] P. Deligne: Letter to E. Looijenga, 9/3/1974. Available at: http://homepage.univie.ac.at/christian.stump/Deligne_Looijenga_Letter_09-03-1974.pdf

[4] J. Dénes: The representation of a permutation as the product of a minimal number of transpositions and its connection with the theory of graphs. Magyar Tud. Akad. Mat. Kutató Int. Közl. 4 (1959), $63-71$.

[5] R. Du and F. Liu: Factorizations of cycles and multi-noded rooted trees. Graphs Combin. 31(3) (2015), 551-575

[6] V. Féray and I. Goulden: A multivariate hook formula for labelled trees. J. Combin. Theory A 120(4) (2013), 944-959.

[7] D. Foata and M.-P. Schützenberger: Théorie géométrique des polynômes Eulériens. Lecture Notes in Mathematics, 138, Berlin, Springer-Verlag, 1970.

[8] J. Irving: Minimal transitive factorizations of permutations into cycles. Canad. J. Math. 61(5) (2009), 1092-1117.

[9] M. Josuat-Vergès: Refined enumeration of noncrossing chains and hook formulas. Ann. Combin. 19(3) (2015), 443-460.

[10] C. Krattenthaler and T. Müller: Decomposition numbers for finite Coxeter groups and generalised non-crossing partitions. Trans. Amer. Math. Soc. 362 (2010), 2723-2787.

[11] G. Kreweras and Moszkowski: Tree codes that preserve increases and degree sequences. Disc. Math. 87(3) (1991), 291-296.

[12] A. Postnikov: Permutohedra, Associahedra, and Beyond. Internat. Math. Res. Notices 2009(6), 1026-1106.

[13] C. M. Springer: Factorizations, trees, and cacti. In Eighth International Conference on Formal Power Series and Algebraic Combinatorics, University of Minnesota, June 25-29, pp. 427-438, 1996.

[14] R.P. Stanley: A survey of alternating permutations. Contemp. Math. 531 (2010), 165-196. 